Jhon Elkis Forero-Medina

DOI $10.35381 / \mathrm{cm} \cdot \mathrm{v} 6 \mathrm{i} 11.328$

\title{
Aprendizaje tecnológico para la enseñanza de las matemáticas
}

\section{Technological learning for the teaching of mathematics}

\author{
Jhon Elkis Forero-Medina \\ jhoelfm@gmail.com \\ Institución Educativa Nelson Mandela, Valledupar \\ Colombia \\ https://orcid.org/0000-0003-1977-3970
}

Recibido: 30 de enero de 2020

Revisado: 15 de marzo de 2020

Aprobado: 01 de abril de 2020

Publicado: 30 de abril de 2020

\begin{abstract}
RESUMEN
Se analizó el aprendizaje tecnológico para la enseñanza de las matemáticas en la Institución Educativa Bello Horizonte del Municipio de Valledupar Departamento del Cesar - Colombia. A su vez, se fundamentó en los postulados teóricos de Urribarrí (2005), UNESCO (2006). Metodológicamente se enmarcó como cuantitativa, descriptiva, con un diseño de campo, transeccional y no experimental. La población estuvo constituida por (97) sujetos. Se utilizó la técnica de la observación mediante encuesta, siendo elaborado un instrumento tipo cuestionario el cual constó de (24) ítems; validado mediante el juicio de expertos, cuyos resultados se le aplicó el coeficiente Alpha de Cronbach dando como resultado un índice de confiabilidad de 0,96 . Para el análisis de los datos, se utilizó la estadística descriptiva. Se encontró como conclusión general que la institución educativa posee debilidades por parte de los docentes, de acuerdo con la percepción de los estudiantes, en cada una de las dimensiones de la variable objeto de estudio. Por lo que se recomienda una posible intervención de las autoridades de la comunidad educativa.
\end{abstract}

Descriptores: Informática educativa; aprendizaje; enseñanza de las matemáticas; tecnología educacional; proceso de aprendizaje.

\section{ABSTRACT}

He analyzed technological learning for teaching mathematics at the Bello Horizonte Educational Institution of the Municipality of Valledupar Department of Cesar Colombia. In turn, it was based on the theoretical postulates of Urribarrí (2005), 


\author{
CIENCIAMATRIA \\ Revista Interdisciplinaria de Humanidades, Educación, Ciencia y Tecnología \\ Año VI. Vol. VI. N¹1. Julio - Diciembre 2020 \\ Hecho el depósito de ley: pp201602FA4721 \\ ISSN-L: 2542-3029; ISSN: 2610-802X \\ Universidad Nacional Experimental Francisco de Miranda (UNEFM). Santa Ana de Coro. Venezuela
}

\title{
Jhon Elkis Forero-Medina
}

UNESCO (2006). Methodologically it was framed as quantitative, descriptive, with a field design, transectional and not experimental. The population consisted of (97) subjects. The observation technique was analyzed through a survey, and a questionnaire-type instrument was prepared, which consists of (24) items; validated by expert judgment, whose results were applied with the Cronbach's alpha coefficient, resulting in a reliability index of 0.96 . For data analysis, analyze descriptive statistics. It was found as a general conclusion that the educational institution has weaknesses $\mathrm{n}$ the part of the teachers, according to the perception of the students, in each of the dimensions of the variable under study. Therefore, a possible intervention by the authorities of the educational community is recommended.

Descriptors: Educational computing; learning; mathematics education, educational technology; learning processes.

\section{INTRODUCCIÓN}

El desafío para las organizaciones en general y las educativas en particular es cómo aprovechar el conocimiento creado por los individuos, y ampliarlo para que se transforme en conocimiento de la organización dedicada a las actividades educativas.

Es ahí donde radica la importancia del proceso de aprendizaje tecnológico, como una herramienta que permite a las instituciones educativas transmitir el conocimiento desde el nivel del individuo, al nivel de la institución, y convertirlo en una ventaja competitiva, por ende, la razón de este artículo es darle la mayor importancia y aplicabilidad al mismo.

En tal sentido, las tecnologías de la información y comunicación (TIC) juegan un papel importante que experimenta la actual sociedad, en particular en lo que respecta al aspecto educativo. Para ello, se hace necesario que el docente realice planteamiento de opciones tecnológicas para el proceso de enseñanza - aprendizaje; y la institución apoye tales incitativas para todas las actividades, especialmente para ser aplicadas a todas las asignaturas y particularmente las del área numérica como las matemáticas. Por lo anterior, los docentes recurren a los recursos tecnológicos para la enseñanza de las matemáticas, en virtud de que sus estrategias deben ser orientadas por el maestro. De esta forma, los estudiantes puedan aprovechar también al máximo estas herramientas, logrando aprendizajes significativos para la construcción de su conocimiento en lo conceptual y en lo procedimental. 
Por otra parte, es importante señalar que el aprendizaje tecnológico para la enseñanza de las matemáticas en la Educación Básica Secundaria y Media, requiere de cierta didáctica enmarcada en la globalización del conocimiento, pudiendo ser fortalecida a través de las herramientas ofrecidas por el Internet. Desde la plataforma de Internet se puede acceder a información teórica - práctica, que ayuden al estudiante a la construcción de contenidos de esta asignatura tan importante dentro del pensum curricular, por su utilidad en la vida diaria, el aprendizaje de otras asignaturas y habilidades de pensamiento. Es así entonces como es importante resaltar según observaciones informales hechas por parte del investigador, como miembros activos de dicha institución con larga trayectoria en el quehacer educativo y enseñanza de las matemáticas, una situación que dista mucho de lo que debería representar un proceso de enseñanza aprendizaje para la calidad educativa que considere al menos a los profesores y a los estudiantes en instituciones educativo de trayectoria.

\section{DESARROLLO TEÓRICO}

\section{Definiciones de Aprendizaje Tecnológico}

En relación con el Aprendizaje Tecnológico, se revisaron varias teorías y postulados de diversos autores, el cual venido evolucionando paulatinamente. Para ello, se recomienda revisar lo señalado por Arturo Torres Vargas sobre Aprendizaje y Construcción de Capacidades Tecnológicas. En tal sentido, se ha venido hablando de la importancia del aprendizaje en el desarrollo de la tecnología y de la industria en el marco de la firma. En todo caso, siguiendo a Torres Vargas (2006), el aprendizaje puede entenderse como esa variedad de procesos a través de los cuáles los individuos y a través de ellos, las organizaciones adquieren conocimientos y habilidades técnicas (Bell 1984). Sobre este aspecto está orientada esta investigación en el ámbito de las instituciones educativas.

El desafío para las organizaciones es como aprovechar el conocimiento creado por los individuos, y ampliarlo para que se transforme en conocimiento de la organización. Es ahí donde radica la importancia del proceso de aprendizaje tecnológico, como una herramienta que permite a las empresas o instituciones en este caso las educativas, 


\section{Jhon Elkis Forero-Medina}

transmitir el conocimiento desde el nivel del individuo, al nivel de la institución, y convertirlo en una fortaleza y ventaja.

Por tanto, Velásquez (2005) ubica al proceso de aprendizaje tecnológico en el centro de la gestión tecnológica en general, siendo rodeado por un ciclo de actividades de focalización, capacitación, implementación y vigilancia, sin depender directamente de ninguna de ellas, pero afectándolas y potenciándolas a todas.

De la misma manera Vargas (1998) define el termino Aprendizaje Tecnológico como un proceso colectivo de acumulación de conocimientos y experiencias, como la experiencia acumulada de una empresa o como la experiencia individual acumulada a medida que ocurre ella. Se asocia estrechamente a la formación profesional en la organización; es un valor que se agrega al talento humano, incorporado mediante diversas estrategias, relacionado con la modernización tecnológica interna de la institución.

Esta vez Ávalos (1992) asocia el proceso de Aprendizaje Tecnológico como un subproceso derivado de la gerencia de la tecnología, que supone la creación de un sistema destinado a desarrollar y acumular capacidades tecnológicas, asociadas al sistema de producción empleado por la organización y a su razón productiva.

En función de lo expuesto anteriormente y como afirma Urueta (2018) las TIC cada vez están cobrando mayor auge en los espacios educativos. Por sus beneficios y potencialidades, se requiere de su uso en casi cualquier actividad humana. De manera que en las instituciones educativas se ha potenciado su uso como herramienta en la gestión del director, pero también de los docentes y estudiantes.

\section{Tipos de aprendizaje tecnológico}

Cuando corresponde desarrollar esta dimensión se revisaron las teorías de Velásquez (2005) y señala que todos los procesos de aprendizaje tienen su desarrollo en las capacidades y la acumulación de información, los cuales pueden ser definidos como procesos de creación y aprovisionamiento del conocimiento. Lo cual consiste en la transformación del conocimiento en sus diversas formas útiles y aplicables para la resolución de problemas o simplemente para la creación de nuevos aprendizajes. 


\section{Jhon Elkis Forero-Medina}

Siguiendo con este mismo autor, ubica el aprendizaje tecnológico como el centro de la gestión, como una herramienta que permite al individuo a transmitir sus conocimientos en focos de capacitación, implementación y vigilancia. Del mismo modo, esta vez en palabras de Villavicencio (2006), el aprendizaje tecnológico es esa variedad de procesos por los cuales los individuos adquieren conocimientos y habilidades técnicas dentro de las organizaciones. Por otro lado, es la manera primordial mediante el cual las empresas adquieren capacidades para innovar.

Por su parte, Polanco y Flores (2008) resaltan que entre los diferentes tipos de aprendizaje tecnológicos se determina la dirección y la forma del cambio en las cuales se puede ver reflejado el implemento de mejoras en los procesos de producción y en la modificación de los insumos. Una noción relacionada a los procesos de aprendizaje tecnológicos es la llamada transferencia tecnológica siendo entendida como el paso de un conocimiento o técnica que se ha ido desarrollando en diversas organizaciones donde es adoptada y utilizada.

De lo expuesto se tiene que para el investigador, los tipos de aprendizajes tecnológicos son los que determinan el curso de acción de las diferentes estrategias aprendidas durante el tiempo de permanencia; y son ellas las que le permiten mantenerse en ese lugar cuyo conocimiento puede ser adoptado y luego utilizado en los diversos escenarios. Esto implica grados o etapas o fase de aprendizaje en las organizaciones como elemental, intermedio y avanzado, que se describen a continuación

\section{Contenidos de aprendizaje tecnológico}

Los contenidos de aprendizaje son todas aquellas teorías, fundamentos, habilidades y destrezas que se plantean a manera de objetivos generales y específicos, los cuales actúan como indicadores de los conocimientos que deben adquirir una persona como parte de un pensum o programa de estudios. En otras palabras, los contenidos sintetizan lo que el estudiante debería saber o comprender como resultado de su proceso de aprendizaje y están condicionados por la naturaleza de su objeto de estudio y el nivel de escolaridad que cursa. 
De acuerdo con Sandoval, García y Ramírez (2012), los contenidos de aprendizaje tecnológico, se imparten en el entorno escolar cuando la modalidad educativa es presencial; o a través del uso de Internet, en caso de entornos de aprendizaje virtuales (como en el E-learning). Sin embargo, fuera de ese proceso en el que el facilitador imparte el contenido y el estudiante lo recibe, debe existir un espacio en el que se aplican de forma práctica dichos contenidos, mientras que el aprendiz indaga otras informaciones a través de la autoformación. La complementariedad de estos procesos: teoría-práctica-autoformación, es lo que garantiza la adquisición de un aprendizaje significativo de los contenidos tecnológicos.

Aunado a lo anterior, Valdemi y otros (2014) consideran que los contenidos de aprendizaje tecnológico son todos aquellos donde el proceso de enseñanza está basado en el uso del ordenador y entornos de aprendizaje interactivo. En este contexto se le plantean al sujeto aprendiz contenidos multimedia, contenidos instruccionales, objetivos educativos, software instruccional y general; así como personas, organizaciones o eventos referenciados en la educación con apoyo tecnológico. Para que estos contenidos puedan ser asimilados de manera significativa, es necesaria la utilización apropiada de los recursos de aprendizaje tecnológico, así como las estrategias didácticas y pedagógicas que faciliten este proceso.

\section{Estrategias de aprendizaje tecnológico}

En cuanto a las estrategias de aprendizaje tecnológico, éstas se encuentran integradas por una serie de propuestas orientadoras y de largo alcance en el interior de la de las firmas o en las instituciones educativas. En este último caso, son de carácter didáctico y pedagógico que se han venido incorporando en la enseñanza y difusión de las tecnologías de la comunicación e información (TIC). Todo como una forma de disminuir la brecha tecnológica y garantizar un uso más generalizado de estas plataformas en las diferentes áreas o disciplinas que integran los planes de estudio. Estas estrategias se basan en el aprender a ser, a hacer y a convivir, por lo que garantizan una evaluación más integral de los conocimientos adquiridos.

Partiendo de esta fundamentación, Morillo, Medina y Bracho (2009) reconocen que el aprendizaje tecnológico, es una noción que se ha venido incorporando al contexto 


\section{Jhon Elkis Forero-Medina}

educativo para garantizar unas competencias y destrezas necesarias para determinar aspectos como: qué información se necesita; la forma más apropiada de acceder a ella, así como la forma de seleccionarla, comunicarla y utilizarla de modo apropiado. Para lograr este cometido, es necesaria una alfabetización tecnológica previa, de forma que el aprendiz tenga una noción o precedente que le permita reconocer las estrategias que el facilitador aplica y los objetivos específicos que se persiguen a través de cada una de ellas.

Asimismo, Mercado (2008 p 76) refiere que el aprendizaje tecnológico se encuentra constituido por un conjunto de experiencias tecno-productivas que se vienen incorporando en las empresas a lo largo de su trayectoria para constituir su acervo de conocimientos en la materia. En consecuencia, se distinguen dos tipos de aprendizaje tecnológico, "uno de carácter formal si se logra sistematizar dentro de las instancias organizativas de la empresa o informal si depende únicamente de la experiencia del personal técnico y/u obrero, pero manteniendo en ambos casos su carácter incremental y acumulativo".

En opinión de Novell, (2010), y Oberto y Oberto (2015) como estrategias orientadoras atendiendo a los cambios ambientales, los entornos b-learning, E-Learning y Ulearning, tienen el objeto de ubicar al aprendizaje combinado en un contexto intermedio, entre la enseñanza presencial, la enseñanza a distancia con el uso de la tecnología de la información y la comunicación como elemento primordial. Esto posibilita el enriquecimiento del proceso de enseñanza aprendizaje, por lo cual es importante conocer sus características, tanto educativas, organizativas y técnicas, lo que permitirá planear e implementar de una manera adecuada cada modalidad.

\section{Aprendizaje tecnológico para la enseñanza de la Matemáticas}

Seguidamente se hace una síntesis sobre el Aprendizaje Tecnológico para la enseñanza, enfatizando sobre las matemáticas. En ese orden de ideas, la revisión de literatura encontró una orientación en el contexto organizacional en general, la cual según Mercado (2014), el Aprendizaje Tecnológico está definido como el conjunto de experiencias tecno-productivas que las empresas/firma van acumulando durante su conocimiento en materia de producción de bienes y servicios. Ahora bien, según 


\section{Jhon Elkis Forero-Medina}

Mercado, el Aprendizaje Tecnológico puede ser de tipo formal, si se logra sistematizar dentro de las instancias organizativas; o informal, si solo se refiere a la experiencia del personal, ya sea obrero o técnico, pero manteniendo su carácter acumulativo. Por su parte, también se tiene otra orientación que en palabras de Cabero (2007), afirma sobre la utilización de las tecnologías en general y las TIC en particular en los sistemas educativos, ha ido incrementándose en todos los niveles del aprendizaje. Por ello, el aprendizaje tecnológico es una herramienta utilizada para la enseñanza. Esto representa una de las ventajas que se le incorpora a la tecnología; como instrumento para una formación que ofrece la interacción y comunicación de manera sincrónica y asincrónica entre los dicentes y docentes. Asimismo, el aprendizaje tecnológico en sí misma, es una alternativa pedagógica, dinamizadora de la producción del conocimiento.

Asimismo, en correspondencia con esta segunda orientación, según Fernández (2007), el aprendizaje tecnológico para la enseñanza es una modalidad educativa basada en recursos donde se aportarán diversas oportunidades en beneficio a los estudiantes, los docentes y la institución educativa. De acuerdo con el autor, bajo este enfoque los y las estudiantes son capaces de planificar la búsqueda, procesar, recuperar, evaluar la información; frente a lo cual, los profesores deben motivar a sus estudiantes a que sean activos en el aprendizaje y desarrollen habilidades como la creatividad, originalidad y buscar a través de la toma de decisiones, la solución a los problemas en el marco institucional en que se encuentran.

Aunado a lo anterior, el docente debería proporcionarle al estudiante herramientas adecuadas para que ellos puedan seleccionar la información obtenida. Asimismo, Fernández y otros (2008) indican que, el aprendizaje tecnológico mejora en los discentes la captación de información, ya que emplean diversas herramientas a través de una interfaz que no solo entretienen, sino que facilita la búsqueda de información. Actualmente, en la parte educativa el uso de estos recursos tecnológicos, los docentes han dejado ser fuentes de todo conocimiento. En la actualidad el docente ha pasado a ser una guía para el estudiante para facilitarles el uso de recursos y herramientas que necesita para explorar y elaborar nuevos conocimientos. Ahora bien, en tal 
sentido Soto (2014) define el aprendizaje tecnológico como entornos asociados para promover el aprendizaje a partir de estrategias educativas tecnológicas, creando situaciones de enseñanza que estimulen la potencialidad de los estudiantes, Estos ambientes-entornos de aprendizaje tecnológico, ofrecen una serie de opciones a los docentes, favoreciendo un enfoque constructivista donde son los alumnos quienes asumen la mayor parte del trabajo. Esto permite aprovechar todas las facilidades que esos ambientes ofrecen basados en la construcción y búsqueda de nuevos conocimientos.

No obstante ¿que tienen las matemáticas de especial y por qué se debería tener cuidado en su enseñanza-aprendizaje? Parte de las respuestas a esas preguntas las podría sugerir Camilloni (2012 p 97). Este autor expresa que "la enseñanza de las matemáticas tiene como fin esencial brindar la información y las herramientas conceptuales y desarrollar las habilidades cognitivas y las actitudes que permitan a los alumnos que abordan el aprendizaje de estas ciencias".

Para ello, el propósito es que adviertan similitudes y diferencias en el modo de vida de las personas que habitan en localizaciones y épocas diversas, que tomen conciencia de que existen y existieron distintas culturas y que, en consecuencia, distintos pueblos tienen maneras también diferentes de ver el mundo y de construir sus sistemas de creencias. La comprensión de las características de los diferentes modos de vida requiere el conocimiento de las variables naturales, individuales y sociales que interactúan entre sí y de las explicaciones teóricas de su interrelación.

\section{METODOLOGÍA}

El tipo de investigación es de corte cuantitativo, la cual es definida por Hernández, Fernández y Baptista (2014) como aquella que se lleva a cabo siguiendo un proceso sistemático y ordenado de unos pasos además es descriptiva cuyo objetivo general es analizar el aprendizaje tecnológico para la enseñanza de las matemáticas en la I.E. Bello Horizonte. De acuerdo a lo planteado las investigaciones descriptivas según, Chávez (2007 p. 7), son todas aquellas que se orientan en recolectar informaciones relacionadas con el estado real de las personas, objetos, situaciones o fenómenos. Conceptualizan este tipo de investigación como aquella que permite "medir o recoger 
información de manera independiente o conjunta sobre los conceptos o las variables a los que se refieren".

Por su parte también es importante destacar en palabras de Piña(2020) que por tratarse de que la metodología implementada para el desarrollo de este estudio, considera en sus argumentos diferentes factores que involucran al entorno social y junto con este a los individuos, quien en definitiva son los determinantes de las técnicas que se utilizan para el análisis de los datos obtenidos, se considera que hay que analizar cada una de las variables en estudio.

Del mismo modo, el diseño de la investigación es el camino a seguir por el investigador para lograr los argumentos a las preguntas planteadas en el estudio, siendo definido por Hernández y otros (2014), como el plan o estrategia concebida para obtener la información que se requiere en una investigación. Partiendo de esta premisa, este estudio se encuadra en un diseño no experimental, transaccional, de campo.

Es por ello que la investigación no experimental, es definida por Chávez (2007), como aquella que su objeto no se encamina a la manipulación del fenómeno para generar la especificación de la conducta, por lo tanto, sólo describe causas y consecuencias mediante un resultado implícito. Del mismo modo, Hernández y otros (2014) señalan que el diseño no experimental de un estudio se realiza sin manipular, alterar, modificar, inferir o cambiar las condiciones en que se comportan las variables: los fenómenos se observan tal y como se dan en el contexto natural para después analizarlos, describirlo o racionarlos.

En el mismo orden de significaciones, la investigación se identifica con el tipo de diseño transeccional o transversal. Al respecto, Chávez (2007), es una investigación de este tipo, debido a que en la misma los fenómenos son estudiados solo una vez y sin tomar en cuenta a la evolución de tiempo. De igual manera, el diseño se tipifica como de campo, por cuanto los datos para estudiar cada variable se obtienen del mismo lugar donde se presenta la situación objeto de estudio.

En el mismo contexto, Sabino (2008), explica que la investigación es de campo, porque los datos se recogen en forma directa de la realidad es decir en la Institución Educativa Bello Horizonte mediante el trabajo del investigador; es decir, que la 


\section{Jhon Elkis Forero-Medina}

información se extrae directamente de una situación real con miras a presentar resultados, y con el fin de optimizar los procesos llevados a cabo en las mismas.

Es por ello, que para esta investigación en particular la muestra queda constituida por una institución educativa, la cual cuenta con noventa y siete (97) sujetos: específicamente noventa y dos (92) estudiantes de noveno grado los cuales fueron seleccionados por las características particulares que se manejan dentro del contexto investigativo del estudio; y cinco (5) docentes de matemática que prestan sus servicios profesionales en el centro educativo teniendo en cuenta sus características, roles, desempeños y relaciones desarrolladas en el contexto escolar de la Institución Educativa Bello Horizonte.

Otra tarea prioritaria en el enfoque cuantitativo, en cuanto a los instrumentos, es la validación de constructo, la cual se refiere, de acuerdo con Hernández y otros (2014) se refiere al grado en que un instrumento realmente mide el concepto/la variable que se pretende medir: fundamentado en la tabla de operacionalización de la variable y sus relaciones teóricas allí presentes. De modo similar, Méndez (2007), define la validez de constructo como el grado en que una prueba mide lo que el investigador se propone medir. Este aspecto es importante para asegurar al investigador que la información que se obtendrá del instrumento cumplirá su propósito dado el enfoque cuantitativo seguido en esta investigación.

En esta investigación, el cuestionario o instrumento se utilizó una escala de Likert, con un nivel de medición de la variable Aprendizaje tecnológico para la enseñanza de las matemáticas, con cuatro alternativas de respuesta, donde se presenta el Instrumento utilizado fue sometido al proceso de validación), a través del juicio de cinco (5) expertos, con el propósito de evaluar su redacción y contenido; e igualmente verificar la efectividad del mismo en función al logro de los objetivos. Se destaca que estos expertos hicieron sugerencias al instrumento en cuanto a redacción, pertinencia, y/o sustitución de algunas palabras para luego considerarlo como válido.

El análisis de los datos en el enfoque cuantitativo de este estudio, se realizó aplicando la estadística descriptiva, de acuerdo al criterio de Tamayo y Tamayo (2012). Asimismo, el autor citado define la desviación estándar como aquella medida 


\section{Jhon Elkis Forero-Medina}

(cuadrática) de lo que se apartan los datos de su media, y por tanto, se mide en las mismas unidades que la variable; mientras que las medias.

Para ello, se consideró el análisis frecuencial y porcentual de las alternativas seleccionadas por los miembros de la institución (estudiantes-profesores) en el instrumento de recolección de datos. Igualmente, para categorizar los puntajes que derivan de los resultados en cuanto a la variable Aprendizaje Tecnológico para la enseñanza de las matemáticas, se consideró el siguiente baremo (Cuadro 6) de medición elaborado por el autor, el cual incluye las medias y las desviaciones estándar de los valores obtenidos.

Así, para proceder al análisis de los datos, se realizó la tabulación de estos con el empleo de tablas, dentro de estas se asentaron los códigos de respuestas que emitieron los integrantes de la población (docentes y estudiantes) objeto de estudio. Seguidamente, para el análisis de los datos se utilizó el paquete computarizado SPSS versión 20.0, a través de los criterios de la estadística descriptiva, en lo que respecta a un análisis con tablas de frecuencia, porcentajes, y la determinación del promedio o media aritmética. De igual manera, para categorizar los puntajes se elaboró el siguiente baremo por parte del investigador para las variables seleccionadas.

\section{RESULTADOS}

A continuación, se presenta la tabla 1, la misma muestra las respuestas de forma porcentual obtenidas luego de administrar el instrumento de recolección de datos a las unidades de análisis definidas para la presente investigación, así como el promedio por alternativa de respuesta y medias aritméticas para la variable Aprendizaje Tecnológico para la Enseñanza de las Matemáticas, distribuido los referidos valores entre las dimensiones que conforman a la variable: 
Jhon Elkis Forero-Medina

Tabla 1

Distribución de Frecuencia de la Variable Aprendizaje Tecnológico para la Enseñanza de las Matemáticas

\begin{tabular}{|c|c|c|c|c|c|c|c|c|c|c|c|c|c|c|c|c|c|c|c|c|c|c|}
\hline \multirow{4}{*}{ Dimensiones } & \multicolumn{22}{|c|}{ Alternativas de Respuestas } \\
\hline & \multicolumn{4}{|c|}{ Siempre } & \multicolumn{4}{|c|}{ Casi Siempre } & \multicolumn{4}{|c|}{ Casi Nunca } & \multicolumn{4}{|c|}{ Nunca } & \multicolumn{4}{|c|}{ Totales } & \multicolumn{2}{|c|}{ Media } \\
\hline & \multicolumn{2}{|c|}{ Doc. } & \multicolumn{2}{|c|}{ Est. } & \multicolumn{2}{|c|}{ Doc. } & \multicolumn{2}{|c|}{ Est. } & \multicolumn{2}{|c|}{ Doc. } & \multicolumn{2}{|c|}{ Est. } & \multicolumn{2}{|c|}{ Doc. } & \multicolumn{2}{|c|}{ Est. } & \multicolumn{2}{|r|}{ Doc. } & \multicolumn{2}{|c|}{ Est. } & \multirow{2}{*}{ Doc. } & \multirow{2}{*}{ Est. } \\
\hline & $\mathbf{F a}$ & $\mathbf{F r}$ & $\mathrm{Fa}$ & $\mathbf{F r}$ & $\mathrm{Fa}$ & $\mathbf{F r}$ & $\mathrm{Fa}$ & $\mathbf{F r}$ & $\mathbf{F a}$ & $\mathbf{F r}$ & $\mathrm{Fa}$ & $\mathbf{F r}$ & $\mathbf{F a}$ & $\mathbf{F r}$ & $\mathrm{Fa}$ & $\mathbf{F r}$ & $\mathrm{Fa}$ & $\mathbf{F r}$ & $\mathbf{F a}$ & $\mathbf{F r}$ & & \\
\hline Tipos de Aprendizaje Tecnológico & 3 & $60,00 \%$ & 3 & $3,26 \%$ & 2 & $40,00 \%$ & 19 & $20,65 \%$ & 0 & $0,00 \%$ & 55 & $59,78 \%$ & 0 & $0,00 \%$ & 15 & $16,30 \%$ & 5 & $100 \%$ & 92 & $100 \%$ & 3,44 & 2,08 \\
\hline Contenido de Aprendizaje Tecnológico & 3 & $60,00 \%$ & 4 & $4,35 \%$ & 2 & $40,00 \%$ & 28 & $30,43 \%$ & 0 & $0,00 \%$ & 47 & $51,09 \%$ & 0 & $0,00 \%$ & 13 & $14,13 \%$ & 5 & $100 \%$ & 92 & $100 \%$ & 3.27 & 2,24 \\
\hline Estrategia de Aprendizaje Tecnológico & 3 & $60,00 \%$ & 7 & $7,61 \%$ & 2 & $40,00 \%$ & 27 & $29,35 \%$ & 0 & $0,00 \%$ & 39 & $42,39 \%$ & 0 & $0,00 \%$ & 19 & $20,65 \%$ & 5 & $100 \%$ & 92 & $100 \%$ & 3.53 & 2,23 \\
\hline Promedio Unid.Análisis & & $60,00 \%$ & & $5,07 \%$ & & $40,00 \%$ & & $26,81 \%$ & & $0,00 \%$ & & $51,09 \%$ & & $0.00 \%$ & & $17,03 \%$ & & $100,00 \%$ & & $100 \%$ & 3,41 & 2,18 \\
\hline \% Orientación de la Frecuencia & \multicolumn{8}{|c|}{$32,97 \%$} & \multicolumn{8}{|c|}{$17,03 \%$} & \multirow{2}{*}{\multicolumn{4}{|c|}{ Mediana }} & \multirow{2}{*}{ Alta } & \multirow{2}{*}{ Ваја } \\
\hline Orientación de la Frecuencia & & & & Favo & rab & & & & & & & Desfay & vorab & & & & & & & & & \\
\hline Media de la Variable & \multicolumn{22}{|c|}{2,80} \\
\hline Categorización de la Varaible & \multicolumn{22}{|c|}{ Medianamente Adecuada } \\
\hline
\end{tabular}

Fuente: Instrumento de recolección de datos (2020)

En la tabla 1, se puede observar el promedio porcentual del resultado de las unidades de análisis por alternativa de respuesta, en la cual se puede apreciar que la alternativa con mayor puntaje es siempre con un $60 \%$ desde la percepción de los docentes, seguida de casi siempre con un $40 \%$. Las alternativas casi nunca y nunca no obtuvieron puntaje. Desde el punto de vista los estudiantes, la alternativa de respuesta con mayor frecuencia de selección la presenta la alternativa casi nunca con un 51,09\% de preferencia, seguida de la alternativa casi siempre con un $26,81 \%$, luego nunca con $17,03 \%$ y finalmente siempre con solo un $5,07 \%$.

En base a lo anterior, en la tabla 4 cuatro también se puede observar la orientación de las alternativas de respuestas en función del análisis frecuencial tomando en cuenta respuestas de docentes y estudiantes. En este sentido, la orientación "Favorable" presenta el mayor puntaje con $32,97 \%$ versus una orientación "Desfavorable" con un 17,03\%, significando esto una tendencia "Mediada" de acuerdo al baremo.

En cuanto a las medias aritméticas para la variable objeto de estudio, la tabla 4 muestra una media de 3,41 relativa a los docentes indicando una tendencia "Alta" y para los estudiantes 2,18, tendencia "Baja", categorizándose según el baremo establecido para el análisis de "Adecuada" en el primer caso y "Poco Adecuada" en el segundo caso, siendo consistentes estos resultados con los arrojados por las distribuciones de frecuencias, tanto para los docentes como para los estudiantes. 


\section{Jhon Elkis Forero-Medina}

De igual manera, se observa la media de la variable la cual presenta un valor de 2,80 categorizándose según el baremo diseñando para la interpretación de los resultados como "Medianamente Adecuada", significando esto que el Aprendizaje Tecnológico para la Enseñanza de las Matemáticas, como variable objeto de estudio es medianamente identificada por la población objeto de análisis, por lo que se deduce la existencia de oportunidades de mejoras en cuanto a su aplicación en las instituciones educativas sujetas a escrutinio en la presente investigación.

De esta manera, se evidencian diferencias significativas entre las respuestas de los docentes y estudiantes, afirmando estos últimos que los docentes casi nunca ponen en práctica el aprendizaje tecnológico para la enseñanza de las matemáticas en la Institución Educativa Bello Horizonte.

Asimismo, en cuanto a la tendencia de las dimensiones de la variable relativa a sus respectivas medias, se puede observar que la dimensión con mayor tendencia de aplicación en la Institución Educativa Bello Horizonte, la presenta Estrategia de Aprendizaje Tecnológico con una media de 3,53 desde la perspectiva de los docentes, seguida de Tipos de Aprendizaje Tecnológico con una media de 3,44, luego Contenido de Aprendizaje Tecnológico con una media iguala 3,27. Por otra parte, desde la percepción de los estudiantes, los mismos consideraron que la dimensión con mayor tendencia de aplicación en la institución antes referenciada es Contenidos de Aprendizaje con una media de 2,24; seguida de Estrategia de Aprendizaje Tecnológico y luego Tipos de Aprendizaje Tecnológico con 2,08.

Cabe destacar que el resultado obtenido de las respuestas de los estudiantes contradice la de los docentes por cuanto la tenencia de las dimensiones desde su punto de vista es "Baja" con una categorización de "Poco Adecuada", según el baremo definido para el análisis de interpretación de los resultados. En consecuencia, los hallazgos no se corresponden con los referentes teóricos de Soto (2014), quien define el aprendizaje tecnológico como entornos asociados para promover el aprendizaje a partir de estrategias educativas tecnológicas, creando situaciones de enseñanza que estimulen la potencialidad de los estudiantes. 


\section{CONCLUSIONES}

Luego del análisis y discusión de los resultados, a continuación, se presentan las conclusiones que se derivan de los hallazgos encontrados tomando en cuenta los objetivos de la investigación:

En relación al primer objetivo específico planteado, se identificaron los tipos de aprendizaje tecnológico para la enseñanza de las matemáticas en la I.E. Bello Horizonte. En tal sentido se tiene que los tipos de aprendizaje tecnológico elemental, intermedio y avanzado, los docentes de estas instituciones expresaron que siempre hacen uso de los tipos de aprendizaje tecnológico para la enseñanza de las matemáticas en la institución educativa en contexto.

Sin embargo, los estudiantes afirmaron que los docentes casi nunca aplican tipos de aprendizaje tecnológico para la enseñanza de las matemáticas, observándose una marcada diferencia entre sus respuestas. Tal afirmación se deriva de la manifestación de los estudiantes en cuanto a que casi nunca se valen de sus conocimientos previos para asimilar la información recibida, con la finalidad de ponerla en práctica, almacenarla y recuperarla al momento de ser necesaria.

Además, nunca logran identificar o discriminar un concepto o una noción de otra, ni realizan inferencias básicas en torno al conocimiento impartido por el docente y; tampoco puede extraer las características de las temáticas estudiadas, sin que sea necesario identificarlas por el docente. Así como tampoco, pueden ejecutar sus trabajos de matemáticas para su posterior revisión con el docente y se les dificulta seleccionar la técnica más apropiada para la realización de la tarea, sobre todo si tienen diferentes alternativas para la solución de los problemas.

En relación al segundo objetivo específico formulado, se categorizaron los contenidos de aprendizaje tecnológico para la enseñanza de las matemáticas en la I.E. Bello Horizonte. En tal sentido se tiene que los contenidos de aprendizaje tecnológico, conceptuales y procedimentales, los docentes de estas instituciones expresaron que siempre ponen práctica de los contenidos de aprendizaje tecnológico para la enseñanza de las matemáticas en la institución educativa en contexto. Sin embargo, los estudiantes afirmaron que los docentes casi nunca hacen uso de los contenidos 
de aprendizaje tecnológico para la enseñanza de las matemáticas, observándose discrepancia entre sus respuestas.

Esta conclusión se fundamenta en la percepción de los estudiantes en cuanto a que casi nunca ponen en práctica las nociones y teorías previas necesaria para aplicar los conocimientos en el manejo y operación de dispositivos tecnológicos en las matemáticas. Además, tampoco aplican los fundamentos adquiridos de las matemáticas a través del uso de programas computacionales especializado en la asignatura.

En cuanto a lo planteado en el tercer objetivo específico, se describieron los aspectos estratégicos de aprendizaje tecnológico en las matemáticas en la I.E. Bello Horizonte, en consecuencia, se tiene que los aspectos estratégicos de aprendizaje tecnológico en este caso e-learning, b-learning y u-learning, los docentes de esta institución expresaron que siempre hacen uso los aspectos estratégicos de aprendizaje tecnológicos en las matemáticas durante el proceso de enseñanza de la asignatura. Sin embargo, los estudiantes afirmaron que los docentes casi nunca hacen uso de estos aspectos estratégicos en la enseñanza de las matemáticas, observándose discordancia entre sus puntos de vistas.

Tal afirmación se deriva de la percepción de los estudiantes en cuanto a que, casi nunca hacen uso de las tecnologías de la comunicación e información como materiales y recursos multimedia para interactuar con el docente, ni con sus pares en el aprendizaje de las matemáticas. Así mismo manifestaron que casi nunca, los docentes ponen en práctica la enseñanza de las matemáticas utilizando como estrategia de aprendizaje tecnológico el b-learning. De igual manera, tampoco hacen uso de medios electrónicos para desarrollar las tareas de matemáticas, ni en la escuela, ni fuera de ella. Además, nunca el docente utiliza la creación de blogs como estrategia del aprendizaje tecnológico y tampoco hacen uso de los softwares educativos como estrategia del aprendizaje tecnológico en el aula de clases.

Finalmente, en cuanto al objetivo general, se analizó el aprendizaje tecnológico para la enseñanza de las matemáticas en la I.E. Bello Horizonte, se puede concluir, que la institución educativa en estudio, se detectaron debilidades por parte de los docentes, 


\section{Jhon Elkis Forero-Medina}

de acuerdo con la percepción de los estudiantes, en cada una de las dimensiones de la variable objeto de estudio. Por lo que representa una posible intervención de las autoridades de la comunidad educativa.

De igual manera se detectó una posición bastante discordante en relación a los docentes con los estudiantes por cuanto se niegan a el uso de las tecnologías en esta área de Matemática por considerarla que ellos la pueden manejar de manera práctica sin el uso de ellas; sin embargo, los estudiantes se les hace un tanto complejo entender los contenidos expuestos por ellos.

\section{RECOMENDACIONES}

En consideración a las conclusiones obtenidas, se da paso a las recomendaciones en función de los aspectos relevantes de acuerdo a los puntajes que, según el investigador, requieren por parte de las instituciones objeto de estudio una posible intervención. En este sentido, se recomienda ejecutar las siguientes acciones, para dar solución a corto y mediano plazo a las problemáticas descritas al inicio de la investigación; se recomienda lo siguiente:

Fortalecer los tipos de aprendizaje tecnológico en los docentes, con la finalidad de hacer uso de los mismos en el proceso de enseñanza de las matemáticas, para así lograr los objetivos instruccionales en sus estudiantes a través de la tecnología de información y comunicación (TIC). En tal sentido, se recomienda la transformación del conocimiento en sus diversas formas útiles y aplicables para la resolución de problemas o simplemente para la creación de nuevos aprendizajes en el estudio de las matemáticas.

Para ello es necesario hacer uso de los tipos de aprendizaje tecnológico (elemental, intermedio y avanzado), como una herramienta que permite al docente a transmitir sus conocimientos en focos de capacitación, implementación y vigilancia; de esta manera, los estudiantes adquirirán conocimientos y habilidades técnicas, dentro y fuera de la institución educativa.

Promover los contenidos de aprendizaje tecnológicos en la enseñanza de las matemáticas, con la finalidad de que el estudiante logre asimilar significativamente los conocimientos básicos y elementales de la asignatura, para los cual se recomienda a 
los docentes ampliar los contenidos conceptuales para que el estudiante pueda englobar conceptos, hechos, teorías y principios de las matemáticas.

Así mismo, se debe profundizar en los contenidos procedimentales, con el objeto que el estudiante una vez adquirido los conceptos o nociones de las matemáticas, tiene los fundamentos necesarios para ejecutar las acciones prácticas que le son planteadas por el docente. A partir de estos contenidos, se fortalecen habilidades intelectuales, motrices, destrezas, estrategias y procesos en los que se ejecutan una serie de acciones para el desarrollo no solo las matemáticas, sino para otras asignaturas.

En relación a la discrepancia entre el uso de las tecnologías para el área de Matemática se recomienda a los docentes la utilidad de las mismas, por cuanto representan un valioso esfuerzo sobre todo con las estrategias empleadas, así los estudiantes pueden aprovechar mejor estas enseñanzas y así hacerla de manera más interactiva.

Finalmente, hacer uso de las estrategias del aprendizaje tecnológico, con la finalidad de ampliar los recursos instruccionales en la enseñanza de las matemáticas, para lo cual se recomienda que el docente, ponga en práctica las diferentes estrategias del aprendizaje tecnológico como los son; e-learning, b-Learnig, y u-Learnig.

Esto con la finalidad de integrar una serie de propuestas de carácter didáctico y pedagógico que se han venido incorporando en la enseñanza y difusión de las tecnologías de la comunicación e información (TIC), como una forma de disminuir la brecha tecnológica y garantizar un uso más generalizado de estas plataformas en las diferentes áreas o disciplinas que integran los planes de estudio, es este caso, las matemáticas. Estas estrategias se basan en el aprender a ser, a hacer y a convivir, por lo que garantizan una evaluación más integral de los conocimientos adquiridos por parte de los estudiantes. 


\section{AGRADECIMIENTOS}

Se agradece especialmente a los docentes y estudiantes del Institución Educativa Bello Horizonte del Municipio de Valledupar Departamento del Cesar - Colombia; quienes colaboraron y apoyaron con la investigación.

\section{REFERENCIAS CONSULTADAS}

Ávalos, I. (1992). Aproximación a la gerencia de la Tecnología en la Empresa. Papers de Trabajo IESA N-16. Venezuela. Ediciones IESA.

Cabero, J (2007) Nuevas tecnologías, comunicación y educación. EDUTEC. Revista Electrónica de Tecnología Educativa, nª 1.

Camilloni, S (2012) "El uso de estrategias metacognitivas en la enseñanza de la matemática". Serie Estudios en Educación Matemática. Sociedad chilena de ciencias naturales a través del análisis de su discurso profesional. Facultad de Educación. Pontificia Universidad Católica de Chile.

Chávez, N (2007) Introducción a la investigación educativa. Pie de Imprenta, Maracaibo: Gráfica González, 2007

Fernández, J y Delavault, D (2008) Software educativos. Educación Médica Superior; 24(1)97-110.

Fernández, R (2007) Competencias profesionales del docente En la sociedad del siglo XXI. Recuperado el 25 de Marzo de 2018 de www.uclm.es/profesorado/ricardo/cursos/competenciaprofesionales.pdf

Hernández, R; Fernández, C y Baptista, P. (2014). Metodología de la Investigación. México, D.F., Mc Graw Hill.

Méndez, C. (2006). Metodología. México, D.F., Editorial Limusa

Mercado, A (2008) Aprendizaje tecnológico y desarrollo socio institucional: la industria química y petroquímica en Brasil y Venezuela. CENDES fundación Polar. Caracas Venezuela.

Morillo, J Medina, P y Bracho, S (2009) Significados e implicaciones de la alfabetización tecnológica en el desarrollo de niños, niñas y adolescentes. Enl@ce: revista Venezolana de Información, Tecnología y Conocimiento, ISSN 1690-7515, Vol. 11, N.․․ 2 . 


\author{
CIENCIAMATRIA \\ Revista Interdisciplinaria de Humanidades, Educación, Ciencia y Tecnología \\ Año VI. Vol. VI. N¹1. Julio - Diciembre 2020 \\ Hecho el depósito de ley: pp201602FA4721 \\ ISSN-L: 2542-3029; ISSN: 2610-802X \\ Universidad Nacional Experimental Francisco de Miranda (UNEFM). Santa Ana de Coro. Venezuela
}

Jhon Elkis Forero-Medina

Novell, M. (2010). The Blended Future of Learning. En Paine, N. \& Masie, E. (Eds.). Learning Perspectives. Contributed by 40 Global learning leader. (92-97).

Oberto, A y Oberto, G (2015) "Gestión social del conocimiento y aprendizaje ubicuo como estrategia”. Articulo. Revista Opción. Vol.32.

Piña-Ferrer, L. (2020). El COVID 19: Impacto psicológico en los seres humanos. Revista Arbitrada Interdisciplinaria de Ciencias de la Salud. Salud y Vida, 4(7), 188-199. doi:http://dx.doi.org/10.35381/s.v.v4i7.670

Polanco, A y Flores, T (2008) Bases para una política de I\&D e innovación de la cadena de valor del maíz, Foro Consultivo de Ciencia y Tecnología (FCCyT), México, 2008.

Sabino, C (2008) El Proceso de Investigación. Ed. Panapo, Caracas, 1992, 216 págs.

Sandoval, García y Ramírez (2012) Libro CIAMTE 2012 Aprendizaje y Mediación Pedagógica con Tecnologías Digitales.CC AttributionLicense.

Soto, J (2014) Propuesta de aplicación de un modelo de Gestión del conocimiento para las entidades del CITMA. Habana: Dirección de Tecnologías de Información y Gestión del Conocimiento (TIGEC).

Tamayo, M. y Tamayo (2012). El proceso de la investigación científica. México: Lumusa.

Torres Vargas, A (2006). Aprendizaje y Construcción de Capacidades Tecnológicas. En J. Technol. Manag. Innov. Volume 1, Issue 5.

Urueta Vélez, L. (2019). Estrategias de enseñanza y el uso de las tecnologías de información y comunicación en las instituciones educativas departamentales en el Municipio Zona Bananera - Colombia. Revista Arbitrada Interdisciplinaria Koinonía, 4(7), 185-201. doi:http://dx.doi.org/10.35381/r.k.v4i7.200

Valdemi, J y García, M (2014) XII Encuentro Internacional de Matemática. Resúmenes de Ponencias. Universidad del Atlántico. Colombia.

Vargas, R. (1998). Reestructuración industrial, educación tecnológica y formación de ingenieros. Trabajo Especial de Grado para Doctorado. México. Universidad Autónoma de Aguascalientes.

Velásquez, G. (2005). Manual de transferencia y adquisición de tecnologías sostenibles. Costa Rica. CEGESTI. 


\section{CIENCIAMATRIA}

Revista Interdisciplinaria de Humanidades, Educación, Ciencia y Tecnología

Año VI. Vol. VI. N¹1. Julio - Diciembre 2020

Hecho el depósito de ley: pp201602FA4721

ISSN-L: 2542-3029; ISSN: 2610-802X

Universidad Nacional Experimental Francisco de Miranda (UNEFM). Santa Ana de Coro. Venezuela

Jhon Elkis Forero-Medina

Villavicencio, D (2006) La transferencia de Tecnología: Un problema de Aprendizaje Colectivo. Argumentos N 10, 11.

(C2020 por el autor. Este artículo es de acceso abierto y distribuido según los términos y condiciones de la licencia Creative Commons Atribución-NoComercial-Compartirlgual 4.0 Internacional (CC BY-NC-SA 4.0)

(https://creativecommons.org/licenses/by-nc-sa/4.0/). 